\title{
Nonlinear analysis of EEG signals at different mental states
}

Kannathal Natarajan*1, Rajendra Acharya U1 ${ }^{1}$, Fadhilah Alias ${ }^{1}$, Thelma Tiboleng ${ }^{1}$ and Sadasivan K Puthusserypady ${ }^{2}$

\author{
Address: ${ }^{1}$ ECE Division, Ngee Ann Polytechnic, 535 Clementi Road, Singapore 599489 and ${ }^{2}$ Department of ECE, National University of Singapore, \\ Singapore 119260 \\ Email: Kannathal Natarajan* - kna2@np.edu.sg; Rajendra Acharya U - aru@np.edu.sg; Fadhilah Alias - s01255253@np.edu.sg; \\ Thelma Tiboleng - s01255274@np.edu.sg; Sadasivan K Puthusserypady - elespk@nus.edu.sg \\ * Corresponding author
}

Published: 16 March 2004

BioMedical Engineering OnLine 2004, 3:7
Received: 18 September 2003

Accepted: 16 March 2004

This article is available from: http://www.biomedical-engineering-online.com/content/3/1/7

(C) 2004 Natarajan et al; licensee BioMed Central Ltd. This is an Open Access article: verbatim copying and redistribution of this article are permitted in all media for any purpose, provided this notice is preserved along with the article's original URL.

\begin{abstract}
Background: The EEG (Electroencephalogram) is a representative signal containing information about the condition of the brain. The shape of the wave may contain useful information about the state of the brain. However, the human observer can not directly monitor these subtle details. Besides, since bio-signals are highly subjective, the symptoms may appear at random in the time scale. Therefore, the EEG signal parameters, extracted and analyzed using computers, are highly useful in diagnostics. This work discusses the effect on the EEG signal due to music and reflexological stimulation.

Methods: In this work, nonlinear parameters like Correlation Dimension (CD), Largest Lyapunov Exponent (LLE), Hurst Exponent (H) and Approximate Entropy (ApEn) are evaluated from the EEG signals under different mental states.

Results: The results obtained show that EEG to become less complex relative to the normal state with a confidence level of more than $85 \%$ due to stimulation.

Conclusions: It is found that the measures are significantly lower when the subjects are under sound or reflexologic stimulation as compared to the normal state. The dimension increases with the degree of the cognitive activity. This suggests that when the subjects are under sound or reflexologic stimuli, the number of parallel functional processes active in the brain is less and the brain goes to a more relaxed state
\end{abstract}

\section{Background}

The electrical activity of a brain measured by Electroencephalogram (EEG) exhibits complex behavior with nonlinear dynamic properties. This behavior takes the form of EEG patterns with different complexities. Considering this, the nonlinear dynamics theory may be a better approach than traditional linear methods in characterizing the intrinsic nature of EEG. The study of nonlinear dynamics and characterization can contribute to the understanding of the EEG dynamics and underlying brain processes and search for its physiological significance. The literature on the study of the application of the nonlinear dynamics theory to analyze physiological signals, shows that nonlinear approaches were used for analysis of heart rate, nerve activity, renal blood flow, arterial pressure, EEG and respiratory signals $[1,2]$. 
The importance of the biological time series analysis, which exhibits typically complex dynamics, has long been recognized in the area of nonlinear analysis. Several features of these approaches have been proposed to detect the hidden important dynamical properties of the physiological phenomenon. The nonlinear dynamical techniques are based on the concept of chaos and it has been applied to many areas including the areas of medicine and biology. The theory of chaos has been used to detect some cardiac arrhythmia such as ventricular fibrillation [3]. Efforts have been made in determining nonlinear parameters for pathological signals and it has been shown that they are useful indicators of pathologies.

Many investigators, for example, Duke et al [4], has proved that complex dynamical evolutions lead to chaotic regimes. In the last thirty years, experimental observations have pointed out that, in fact, chaotic systems are common in nature. A detail of such system is given in by Boccaletti et al. [5]. In theoretical modeling of neural systems, emphasis has been put mainly on either stable or cyclic behaviors. Perhaps chaotic behavior at neural level could be responsible for schizophrenia, insomnia, epilepsy and other disorders [6-8]. In the past wide range of work has been done in understanding the complexities associated with the brain through multiple windows of mathematics, physics, engineering and chemistry, physiology etc [9-11]. During the past, there has been a sustained interest in describing neural processes and brain signals, especially the EEG, within the context of nonlinear dynamics and theory of deterministic chaos [12,13]. Nonlinear dynamics theory opens new window for understanding behavior of EEG. EEG models were proposed by Freeman et al [14] for neocortical dynamics or by Wright et al[15] for chaotic dynamics to meet requirements in neurobiology. In analysis of EEG data, different chaotic measures such as correlation dimension, Lyapunov exponent and entropy are used in recent literature [16-22]. Approximate Entropy of human respiratory movement during eye-closed walking and different sleep stages was studied by Naoto et al[23].

In this work, EEG is recorded for various mental states such as (1) subjects under normal resting state, (2) subjects listening to classical music, (3) subjects listening to rock music and (4) subjects with foot reflexology stimulation. The effect of the music and reflexological stimulation on the EEG signal is studied by evaluating the nonlinear parameters like Correlation dimension, Approximate Entropy, Largest Lyapunov Exponent and Hurst Exponent.

\section{Methods}

EEG signals are being recorded by using BIOPAC equipment with ACQKNOWLEDGE 3.7.2 as data acquisition software [24]. Silver-Silver chloride electrodes were applied following the 10-20 system. The EEG is recorded at 500 samples/sec with a resolution of 12 bits/sample and for a duration of twenty minutes. Then the data is digitally filtered using $1-50 \mathrm{~Hz}$ band pass filter. The EEG is first recorded for the subject under resting condition with eyes closed. In second state, the EEG is recorded with subject listening to classical music and then followed by rock music. According to Voss et al. [25], the classical and rock music exhibit different $1 / \mathrm{f}$ distribution. Here the hypothesis is that the since the two music have different $1 / \mathrm{f}$ distribution they will have different effects on EEG. Finally the EEGs are recorded with subjects under foot reflexologic stimulation. Reflexology is a science, an art and a skill that uses gentle pressure and manipulation of the feet, hands and ears. It deals with the principle that, there are reflex areas in the feet and hands correspond to various glands, organs and parts of the body. EEGs are recorded from 30 subjects (15 boys and 15 girls) with age ranging from $20 \pm 3$ years for 20 minutes. The data is recorded in sequential order with subject (1) under normal resting state, (2) listening to classical music (3) listening to rock music and (4) with foot reflexology stimulation. Time gap of 30 minutes is given between any two successive recordings to ensure that a previous state stimulus doesn't affect the subsequent state EEG.

\section{Analysis}

In this work, we have analyzed the EEGs using various characteristic measures like Correlation Dimension, Lyapunov exponent, Hurst Exponent, and Approximate Entropy. A brief description of the measures is given below.

\section{Correlation Dimension}

The dimension of a graph can give much information about the nature of the system from which it is derived. The dimension of a graph determined by experimental data is therefore very useful to the understanding of the system, particularly for deciding if the system is periodic, chaotic, or noisy. Mathematically, the dimension of any graph that is made of a finite set of data points is zero. The correlation dimension however can be used to approximate the dimension of these types of graphs. It is most often computed from the time series diagram. A time series diagram is a simplified phase space diagram constructed from a single data vector.

In the phase-space plot shown in Figure 1, X-axis represents the EEG signal X[n] and the Y-axis represents the EEG signal after a delay X[n+delay]. The choice of an appropriate delay is calculated using the minimal mutual information technique $[26,27]$. The phase space plot for various mental states is shown in Figure 1. 
Phase-Space Plots

$X^{\prime}(t)=(t)$ us $X^{\prime}(t)$ us $X(t) \quad$ FAZ_EY 1 .DAT

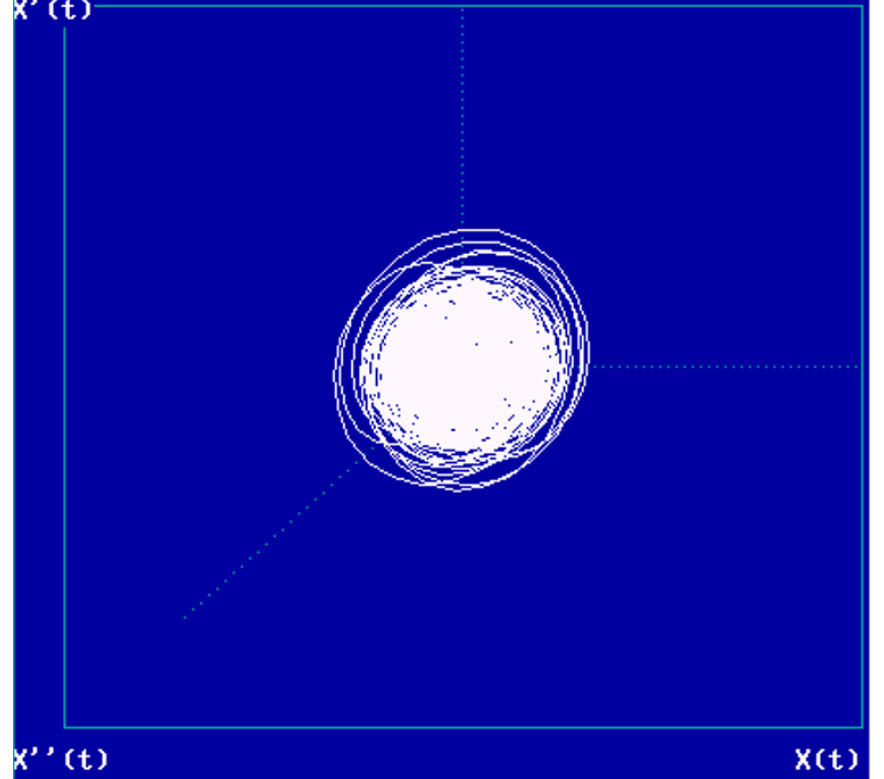

a

Phase-Space Plots

$x^{\prime \prime}(t)$ us $X^{\prime}(t)$ us $x(t) \quad$ FAZ_CL 1.DAT

$x^{\prime}(t)$

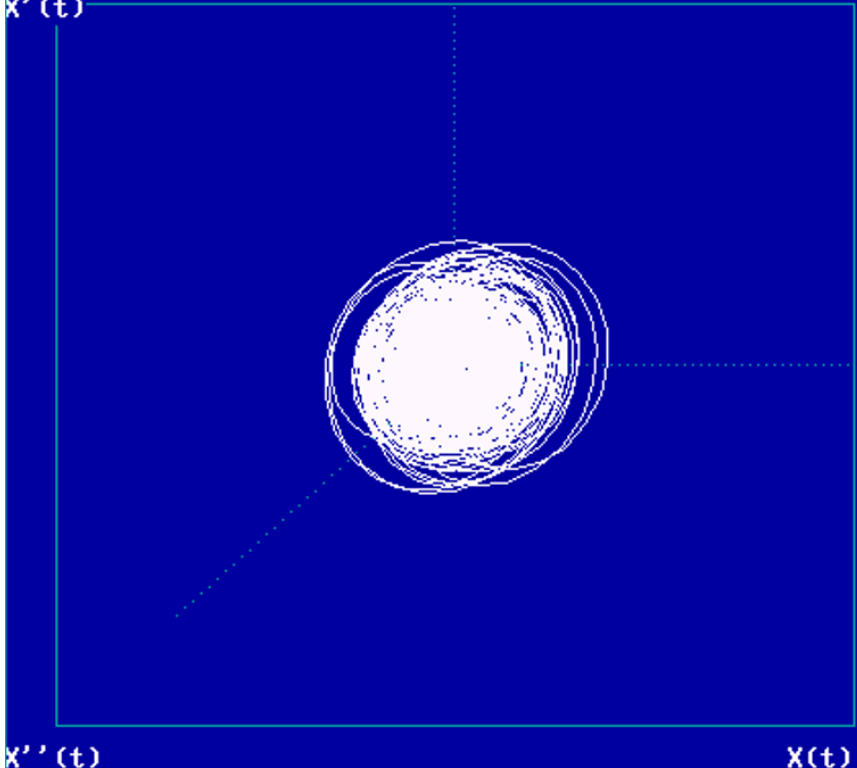

c

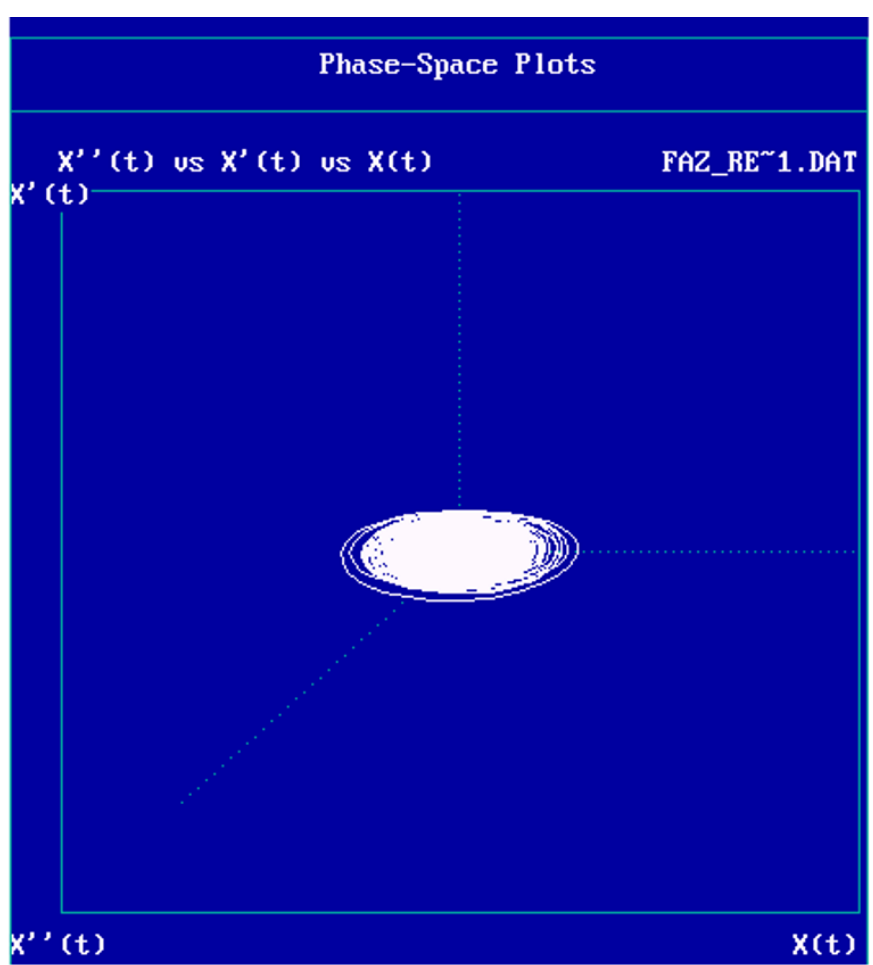

b

Phase-Space Plots

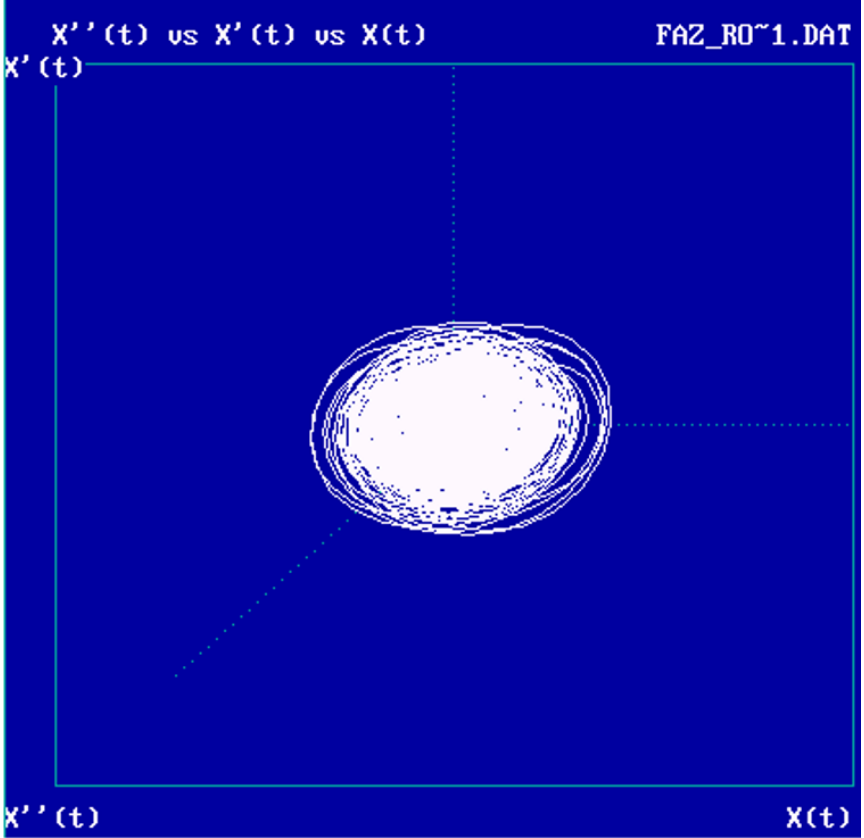

d

Figure I

Phase space plots for various mental states 
Correlation Dimension is one of the most widely used measures of Fractal Dimension. Here we adapt the algorithm proposed by Grassberger and Procaccia $[28,29]$. The idea is to construct a function $C(r)$ that is the probability that two arbitrary points on the orbit are closer together than $r$. This is done by calculating the separation between every pair of $N$ data points and sorting them into bins of width $\mathrm{d} r$ proportionate to $r$. A correlation dimension can be calculated using the distances between each pair of points in the set of $\mathrm{N}$ number of points, $s(i, j)=\left|X_{i}-X_{j}\right|$.

A correlation function, $\mathrm{C}(\mathrm{r})$, is then calculated using,

$\mathrm{C}(\mathrm{r})=\frac{2}{N(N-1)} \sum_{i=1}^{N} \sum_{j=i+1}^{N} \Theta\left(r-\left|X_{i}-X_{j}\right|\right)$

Where $X_{i^{\prime}} X_{j} \rightarrow$ points of the trajectory in the phase space,

$N \rightarrow$ is the number of data points in phase space

$r \rightarrow$ radial distance around each reference point $\mathrm{X}_{\mathrm{i}}$

$\Theta \rightarrow$ is the Heaviside function.

Correlation dimension was calculated using the fundamental definition
$\mathbf{D}_{\text {corr }}=C D=\lim _{r \rightarrow 0} \frac{\log C(r)}{\log (r)}$

The accuracy of the nonlinear time series analysis lies in the selection of optimum embedding dimension [30]. Embedding theorems defined by Takens and Sauer $[31,32]$ states that for a strange attractor of Fractal Dimension $\mathrm{D}$, the embedding using time delay coordinates is one to one if $m \geq 2 D+1$ or $m \geq D_{\text {corr }}$, where $D_{\text {corr }}$ is the correlation Dimension and $\mathrm{m}$ is the embedding dimension. But the limitation on the applicability of the theorems is that the measure $\mathrm{D}$ and $\mathrm{D}_{\text {corr }}$ are not known. For practical applications, it is best to apply the Grassberger and Procaccia algorithm [28] and calculate the $\mathrm{D}_{\text {corr }}$ for various embedding dimension. Then the minimum embedding dimension of the attractor for one to one embedding is $\mathrm{m}+1$, where $\mathrm{m}$ is the embedding dimension above which the $\mathrm{D}_{\text {corr }}$ saturates. In this work, we have calculated $\mathrm{D}_{\text {corr }}$ with an embedding dimension varying from 1 to 10 for all the subjects. The graph of $\mathrm{D}_{\text {corr }}$ Vs Embedding Dimension is shown in Figure 2. From the graph it can be seen that $\mathrm{D}_{\text {corr }}$ saturates after the embedding dimension of 9 . So we have chosen the embedding dimension of $m+1=10$ for analysis. The software used for analysis is CDA Pro Data analyzer [33].

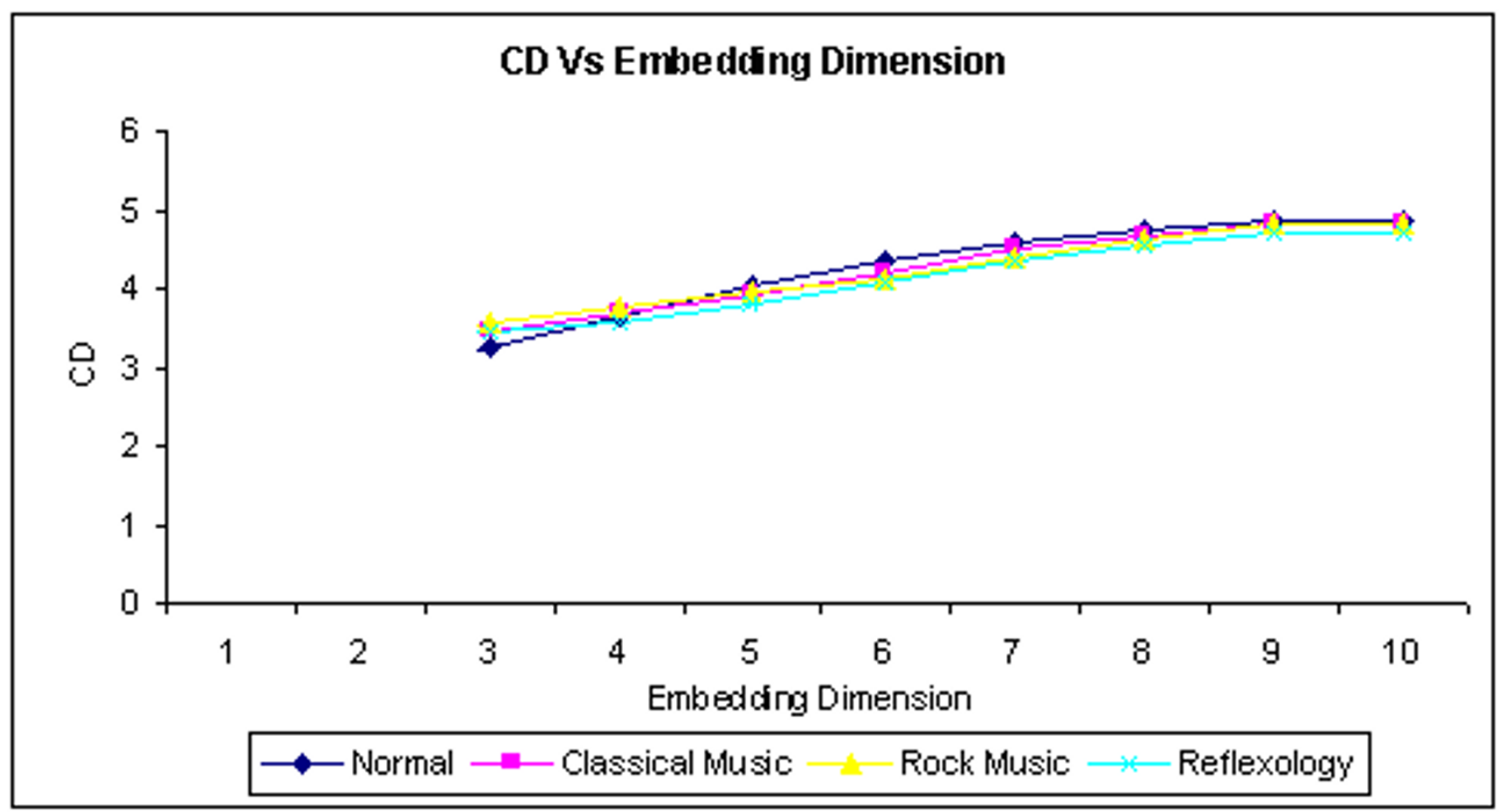

Figure 2

Correlation Dimension Vs Embedding Dimension 
The data points used in calculating the autocorrelation come from a time series, which has too small or too large time resolution may introduce spurious effects. Here, the time resolution is too small, the data may contain multiple copies of essentially the same measurements, which leads to multiple-counting. The correlation dimension is then artificially low because all the points are temporally close to each other. This effect occurs when the time resolution $\Delta$ tof the analyzed data is much smaller than the autocorrelation time $\tau_{a c}$ (or any characteristic time scale).

$\Delta t\left\langle\tau_{a c}\right.$

The correction proposed by Theiler [34] is, for each reference point $X_{i}$ to include measurements $X_{j}$ which are at least $w=\tau_{a c}$ steps away from $X_{i}$

$$
\mathrm{C}(\mathrm{r})=\frac{2}{N(N-1)} \sum_{i=1}^{N} \sum_{j=i+w}^{N} \Theta\left(r-\left|X_{i}-X_{j}\right|\right)
$$

\section{Approximate Entropy}

Entropy is a thermodynamic quantity describing the amount of disorder in the system. From an information theory perspective, the above concept of entropy is generalized as the amount of information stored in a more general probability distribution. Recently a number of different entropy estimators [35-40] have been applied to EEG data to quantify the complexity of the EEG signal. These techniques do not measure the shape of the distribution of the EEG voltages per se, but instead describe how the EEG signals changes with time - either in frequency-space or phase-space. Information about how the EEG signal fluctuates with time is obtained by comparing the time series with itself, but lagged by a specified time interval. This practice is referred as embedding the onedimensional signal in a 'phase-space'. Intuitively it seems sensible that, if an EEG signal is irregular, the position of a particular point will not be easily predicted using knowledge of its previous points; whereas in a regular signal the position of the point will be more reliably predicted. The number of previous (lagged) points used in making the prediction is the embedding dimension $(m)$. For a process whose underlying dimension is $n$ (i.e. which can be described uniquely in terms of $n$ parameters), the required embedding dimension is; $m \geq 2 n+1$, and the required minimum date size to extract these $n$ parameters is $10^{\mathrm{m}}$. It is impractical to properly embed the EEG signal. Thus these techniques are NOT able to fulfill their theoretical promise and extract high-dimensional information from the univariate EEG data-stream. Using these embeddings, the theoretical measure of the rate of "information" generation by a system is the Kolmogorov-Sinai entropy [29]. However this measure diverges to a value of infinity when the signal is contaminated by the slightest noise. Pincus, Gladstone et al. proposed Approximate Entropy (ApEn) as a solution to these problems and successfully applied it to relatively short and noisy data $[41,42]$. The ApEn was used by Bruhn [43] to analyze EEG signals in patients under general anesthesia. According to Steyn-Ross et al [44] the approximate entropy when applied to EEG signals reflects the intra-cortical information flow in the brain. In the calculation ApEn, two parameters $m$ and $r$ must be chosen prior to the computation of ApEn where, $m$ specifies the pattern length, and $r$ is the noise threshold. The ApEn measure is obtained by

$$
\operatorname{ApEn}(m, r, L)=\frac{1}{L-m} \sum_{i=1}^{L-m} \log C_{i}^{m+1}(r)-\frac{1}{L-m+1} \sum_{i=1}^{L-m+1} \log C_{i}^{m}(r)
$$

where $\mathrm{C}_{i}^{m}(\mathrm{r})$ is the correlation integral with embedding dimension $\mathrm{m}$ and time lag of 1.

For this study, $m$ is set to 2 and $r$ is set to $15 \%$ of the standard deviation of each time series. These values are selected on the basis of previous studies indicating good statistical validity for ApEn within these variable ranges [42].

\section{Hurst Exponent $(\mathrm{H})$}

The Hurst Exponent is a measure that has been widely used to evaluate the self-similarity and correlation properties of fractional Brownian noise, the time series produced by a fractional (fractal) Gaussian process. Hurst exponent is used to evaluate the presence or absence of long-range dependence and its degree in a time series. However, local trends (nonstationarities) is often present in physiological data and may compromise the ability of some methods to measure self-similarity. Hurst Exponent is the measure of the smoothness of a fractal time series based on the asymptotic behavior of the rescaled range of the process. In time series analysis of EEG, Hurst Exponent $H$ is used by [45] for characterize the non-stationary behavior of the sleep EEG episodes. The hurst exponent $\mathrm{H}$ is defined as:

$\mathrm{H}=\log (\mathrm{R} / \mathrm{S}) / \log (\mathrm{T})$

where $T$ is the duration of the sample of data and $\mathrm{R} / \mathrm{S}$ the corresponding value of rescaled range. The above expression is obtained from the Hurst's generalized equation of time series that is also valid for Brownian motion. If $\mathrm{H}=$ 0.5 , the behavior of the time-series is similar to a random walk. If $\mathrm{H}<0.5$, the time-series cover less "distance" than a random walk. But if $\mathrm{H}>0.5$, the time-series covers more "distance" than a random walk. $\mathrm{H}$ is related to the fractal dimension $\mathrm{D}$ given by

$\mathrm{H}=\mathrm{E}+1-\mathrm{D}$

where $\mathrm{E}$ is the Euclidean dimension. 


\section{Largest Lyapunov Exponent (LLE)}

Lyapunov Exponent $(\lambda)$ is a quantitative measure of the sensitive dependence on the initial conditions. It defines the average rate of divergence of two neighboring trajectories. An exponential divergence of initially nearby trajectories in phase space coupled with folding of trajectories, to ensure that the solutions will remain finite, is the general mechanism for generating deterministic randomness and unpredictability. Therefore, the existence of a positive $\lambda$ for almost all initial conditions in a bounded dynamical system is widely used definition of deterministic chaos. To discriminate between chaotic dynamics and periodic signals Lyapunov exponent $(\lambda)$ are often used. It is a measure of the rate at which the trajectories separate one from other. The trajectories of chaotic signals in phase space follow typical patterns. Closely spaced trajectories converge and diverge exponentially, relative to each other. For dynamical systems, sensitivity to initial conditions is quantified by the Lyapunov exponent $(\lambda)$. They characterize the average rate of divergence of these neighboring trajectories. A negative exponent implies that the orbits approach a common fixed point. A zero exponent means the orbits maintain their relative positions; they are on a stable attractor. Finally, a positive exponent implies the orbits are on a chaotic attractor.

The algorithm proposed by Wolf et al. [46] is used to Largest LE (LLE) from EEG data. For Given the time series $\mathrm{x}(\mathrm{t})$ for $m$ dimensional phase space with delay coordinate $t$, that is a point on the attractor is given by

$\{x(t), x(t+t), \ldots, x(t+(m-1) t\}$

We locate nearest neighbor to initial point

$\{x(t 0), x(t 0+t), \ldots, x(t 0+(m-1) t\}$

And denote the distance between these two points as $\mathrm{L}(\mathrm{t} 0)$. At a later time $\mathrm{t} 1$, initial length will evolve to length $\mathrm{L}^{\prime}(\mathrm{t} 1)$. The mean exponential rate of divergence of two initially close orbits is characterized by

$$
\lambda=\frac{1}{t_{M}-t_{0}} \sum_{k=1}^{M} \log _{2} \frac{L^{\prime}\left(t_{k}\right)}{L^{\prime}\left(t_{k-1}\right)}
$$

In implementation of this program, the following set of numerical parameters has to be chosen:

$\mathrm{P}=\{\mathrm{m}, \mathrm{t}, \mathrm{T}, \mathrm{Smax}, \mathrm{Smin}, \mathrm{thmax}\}$

where $\mathrm{m}$ is the embedding dimension, $\mathrm{t}$ is delay, $\mathrm{T}$ being evaluation time $(=\mathrm{t} \mathrm{k}+1-\mathrm{t} \mathrm{k}-1)$ and $\mathrm{S}_{\max ^{\prime}} \mathrm{S}_{\min }$ are the maximum and minimum separations of replacement point respectively and $\mathrm{th}_{\max }$ is the maximum orientation error. According to Das et al [47] an embedding dimension between 5 to 20 and a delay of 1 should be chosen when calculating LE for EEG data. In our analysis we have chosen an embedding dimension of 10 and delay of 1 .

\section{Surrogate data}

To test if the attractor geometry and correlation dimension are truly due to chaotic dynamics one must examine these characteristics for surrogate data sets.

The purpose of surrogate data is to test for any nonlinearity in the original data. Nonlinear indexes such as ApEn are computed for several surrogate data series. Their values are compared with that assumed by the nonlinear index computed for the original index [48]. The demonstration of statistically significant difference in ApEn between the original and surrogate data are in keeping with the presence of nonlinear dynamics in the original data.

Surrogate data have Fourier decomposition with the same amplitudes as the empirical data decomposition but with random phase components. This is obtained from the Chaos Data Analyzer.

To test for a statistical significance of difference in original ApEn and the surrogate data, 10 surrogate data series were generated to match each original signal. Then it is subjected to Student $\mathrm{t}$ test distribution. We found that, the surrogate data ApEn and Original data ApEn, are close to each other with confidence level of $95 \%$. The similar procedure is repeated for Correlation Dimension. The surrogate data Correlation Dimension and original data Correlation Dimension are different from each other with confidence level of $93 \%$. Hence the original data contain nonlinear features.

\section{Results}

The phase space plot show the unique pattern for each mental state (Figure 1(a),1(b),1(c) and 1(d)). The correlation dimension parameter $\mathrm{D}_{\text {corr }}$ is estimated for all the experimental data sets and result is shown in Figure 3. It quantifies the variability in a time series. The signal becomes less complex (less random) when the person is subjected music (of his choice) or under reflexological stimulation. Similarly Largest Lyapunov Exponent, Approximate Entropy and Hurst Exponent are calculated for all the data sets are presented in Figure 4, 5, 6. The Figure 4 , depicts the predictability of the signal increases and the signal becomes less chaotic under the influence of music and reflexologic stimulation. Largest Lyapunov Exponent behaves as indicator of long-term behavior. The LLE value closer to 1 indicates the chaotic behavior of the series. This value falls due to the influence of the music and reflexological stimulation. Hence the randomness falls due this external influence. From the Figure 5, it can 


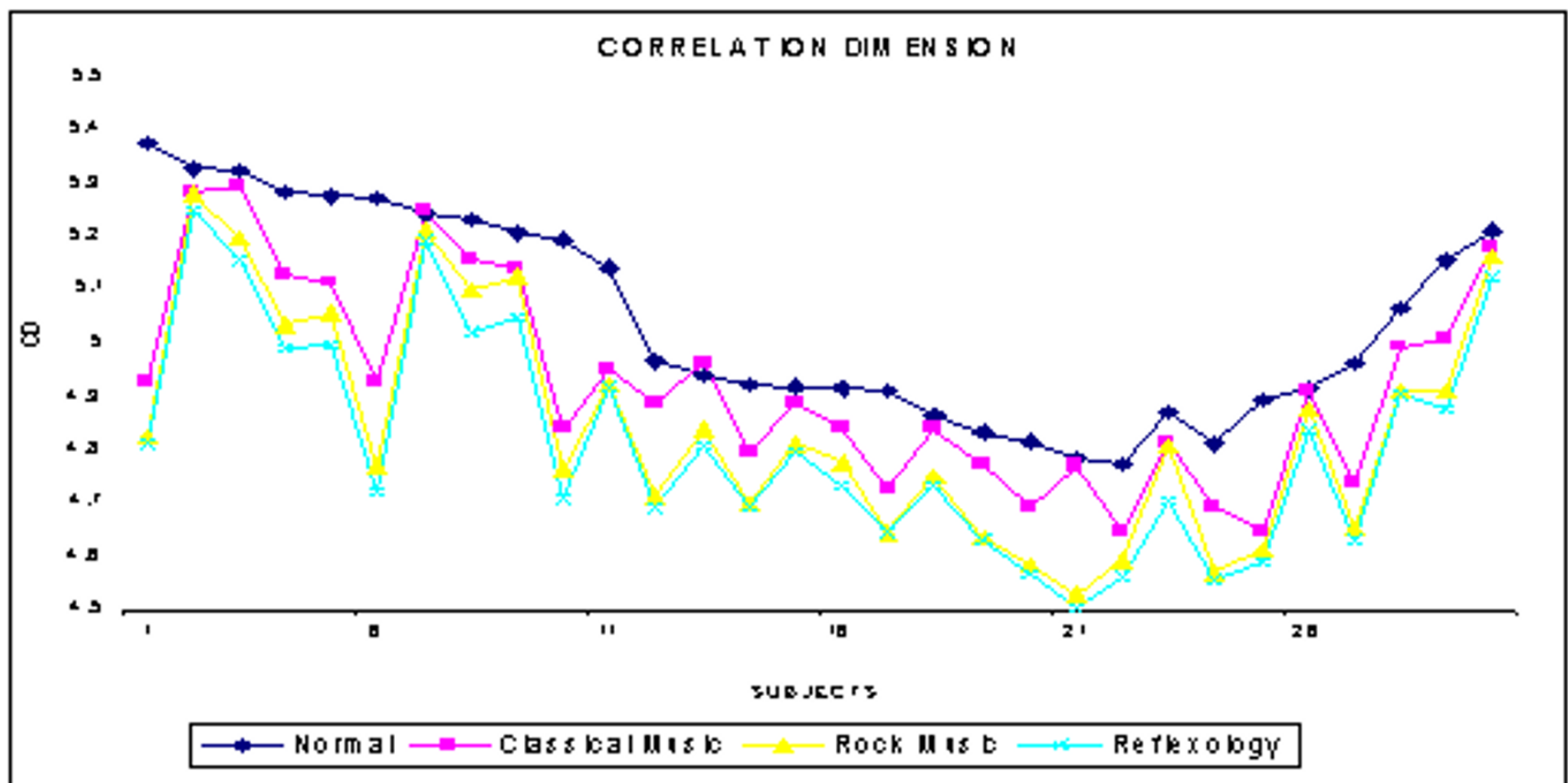

Figure 3

The variation of $C D$ for various mental states

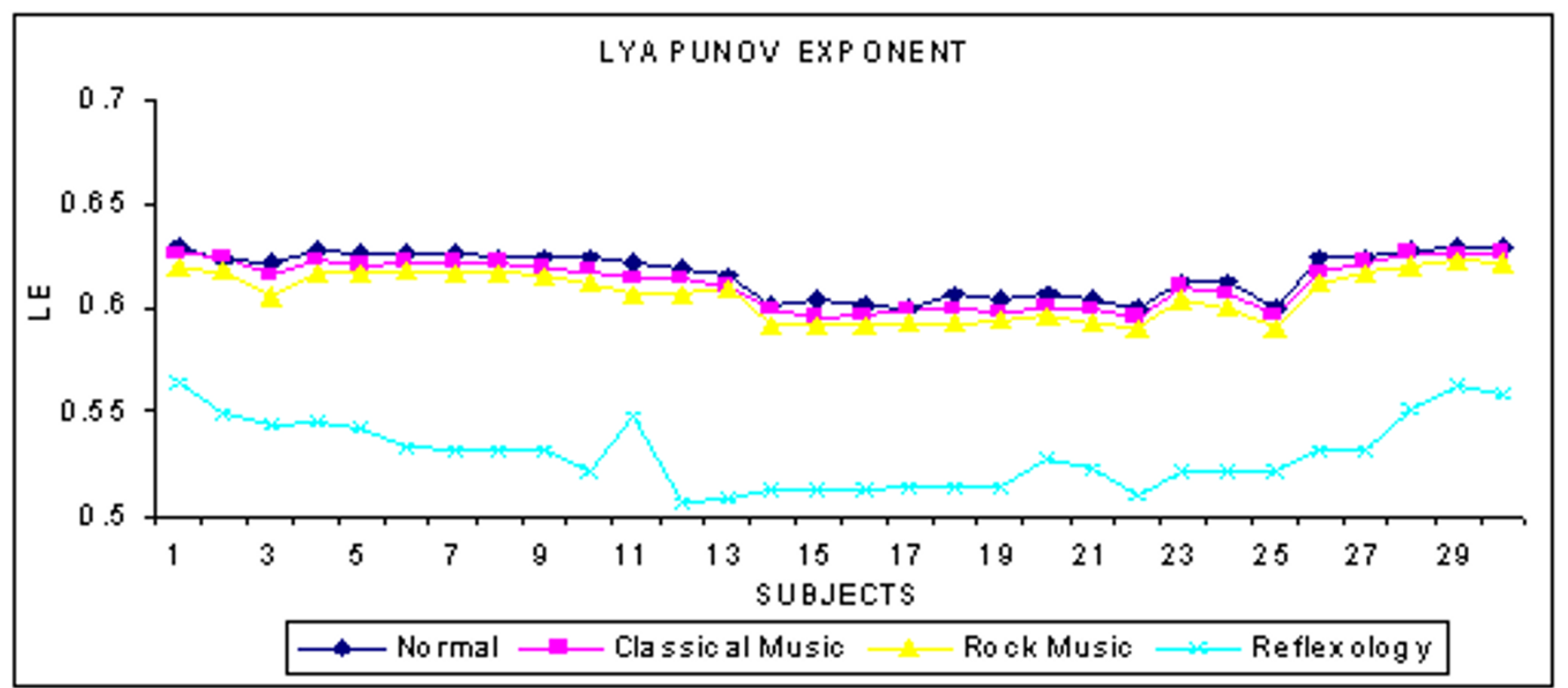

Figure 4

The variation of LLE for various mental states 


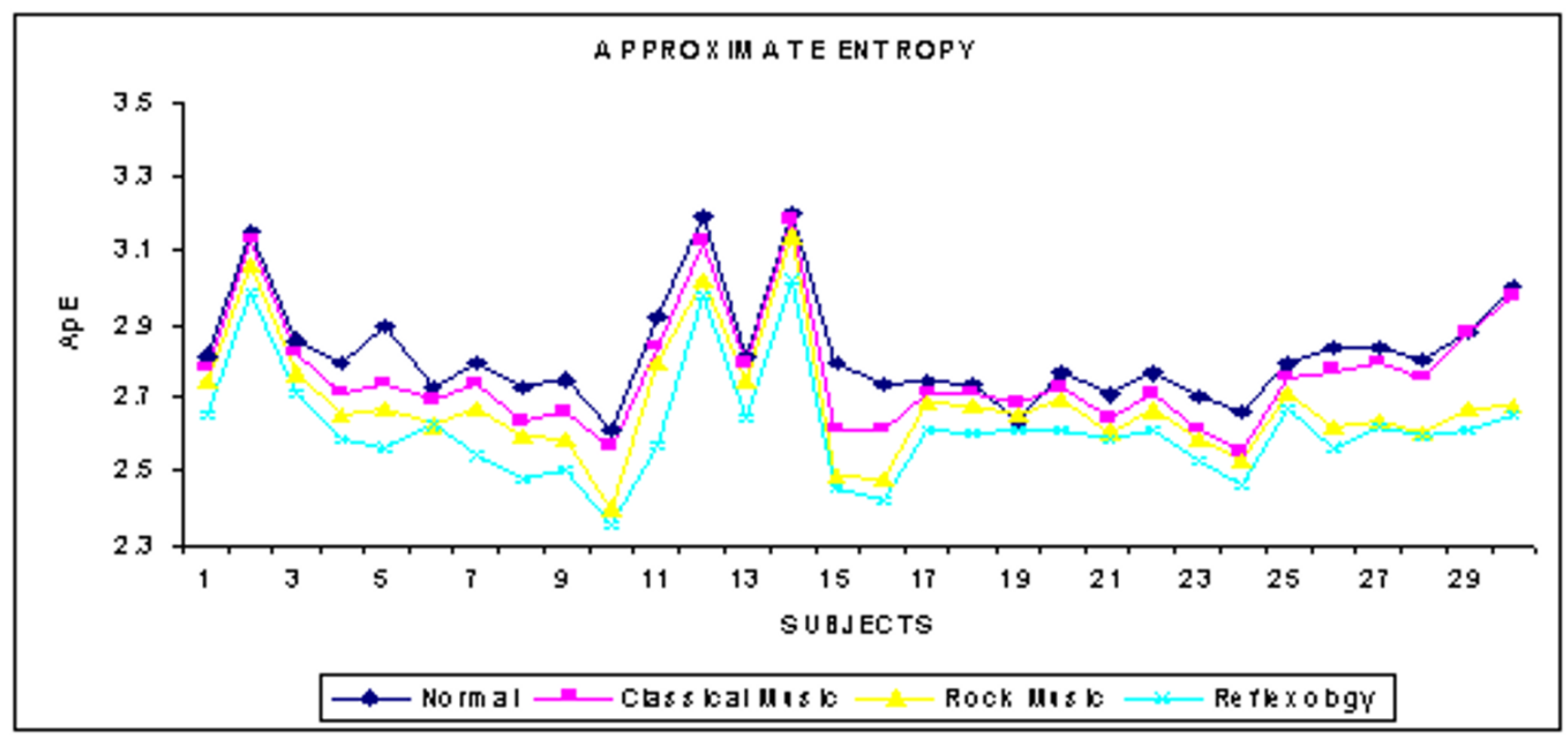

Figure 5

The variation of ApEn for various mental states

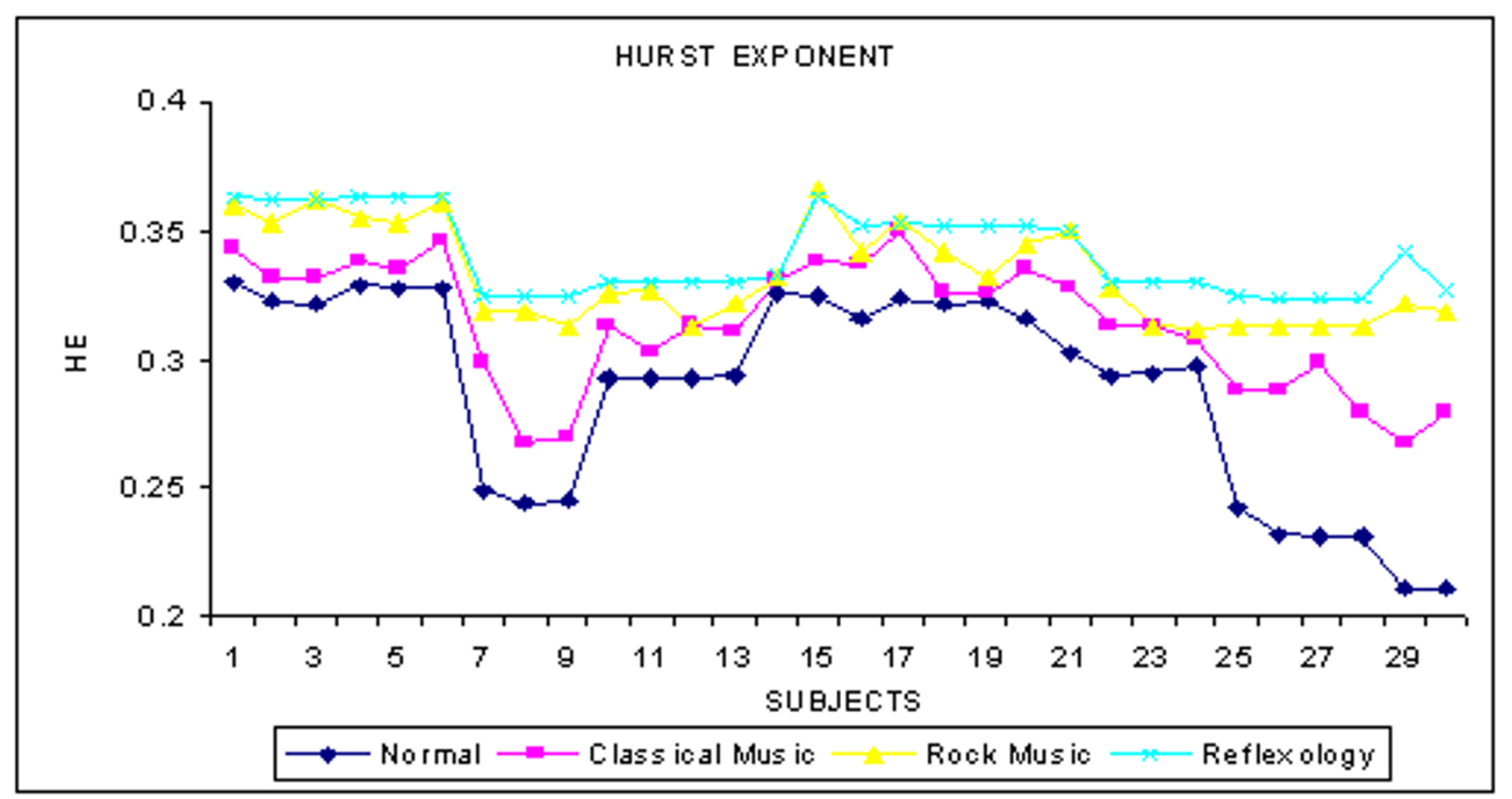

Figure 6

The variation of HE for various mental states 
Table I: Range of nonlinear parameter variation for different mental states

\begin{tabular}{|c|c|c|c|c|c|}
\hline Parameter & Normal resting state & With classical music & With rock music & $\begin{array}{l}\text { With foot reflexologic } \\
\text { stimulation }\end{array}$ & p-value \\
\hline $\begin{array}{l}\text { Correlation } \\
\text { Dimension }\end{array}$ & $5.0426 \pm 0.286$ & $4.921 \pm 0.258$ & $4.846 \pm 0.284$ & $4.828 \pm 0.269$ & 0.0956 \\
\hline Lyapunov Exponent & $0.6185 \pm 0.0107$ & $0.6133 \pm 0.0112$ & $0.6072 \pm 0.0122$ & $0.5297 \pm 0.0231$ & 0.0701 \\
\hline Approximate Entropy & $2.8214 \pm 0.146$ & $2.762 \pm 0.157$ & $2.6833 \pm 0.181$ & $2.615 \pm 0.191$ & 0.1346 \\
\hline Hurst Exponent & $0.2887 \pm 0.0568$ & $0.3133 \pm 0.0432$ & $0.3372 \pm 0.0516$ & $0.3497 \pm 0.0523$ & 0.064 \\
\hline
\end{tabular}

be seen that the same trend is obtained for ApEn. ApEn is the measure of dynamic changes of the EEG signal in time domain. A decrease in entropy indicates higher predictability and a reduced stochastic behavior. The self-similarity parameter, Hurst Exponent $\mathrm{H}$ is obtained for all data sets is shown in Figure 6. If $\mathrm{H}=0.5$ indicates the random behavior of the signal and this value falls under the influence of external stimulus. This means that the randomness decreases due to music/reflexology. The interesting finding is that the randomness or the disorderliness of the EEG is reduced after the subject hears music or reflexologic stimulation. Having computed the values of nonlinear parameters of all the subjects under normal resting condition and under music or reflexologic stimulation, we have performed the statistical analysis on these numeral characteristics. All the results are presented as mean \pm SD with "p" values in Table 1 . The results indicate after the music/ reflexological stimulus, the brain goes relaxed state. In neurophysiology the most cited indicator of relaxation is the rise in alpha wave frequencies will be predominant.

\section{Discussion}

The statistical analysis is performed by comparing the results obtained from various mental states with respect to the normal resting state. Table 1 depicts the statistical outcome of the nonlinear analysis between the normal and other states. It is found that the measures are significantly lower when the subjects are under sound or reflexologic stimulation as compared to the normal state. It is well known that the dimension of EEG time series is closely related to the cognitive activity of the brain [49]. The dimension increases with the degree of the cognitive activity. Our results show that dimension reduces during reflexological stimulation indicating that the brain is not involved in cognitive tasks or thinking rigorously, meaning that the brain is in a stoic, stolid, passive state of relaxation. From the result it can be seen that there is a distinct difference in the correlation dimension in the different mental states with a confidence level of more than $90 \%$. The correlation dimension for the normal group (mean = 5.0426 and $\mathrm{SD}=0.286$ ) is much higher compared to the other groups. A decreased value indicates that the ran- domness of the brain activity is reduced. This suggests that when the subjects are under sound or reflexologic stimuli, the number of parallel functional processes active in the brain is less and the brain goes to a more relaxed state [5055 . This gives rise to the increase in alpha frequencies in the brain waves. The same trend is seen for parameters like Lyapunov exponent and ApEn (LLE ( $\mathrm{p}<0.07)$ ) ApEn ( $\mathrm{p}<$ $0.13)$ and $H(p<0.064))$. From Table 1 it is evident that the nonlinear complexity of the EEG is reduced when subjected to music and reflexolgical stimulus. Hurst exponent $(\mathrm{p}<0.06)$ also shows the same trend because of the very definition of the term Hurst exponent $[\mathrm{H}=\mathrm{E}+1-\mathrm{D}$, where ' $\mathrm{H}$ ' is the Hurst exponent, ' $\mathrm{E}$ ' is the Euclidean dimension and ' $\mathrm{D}$ ' is the correlation dimension]. Hence as ' $\mathrm{D}$ ' decreases ' $\mathrm{H}$ ' has to increase [45]. These results show that the effect of sound and reflexologic stimulation causes EEG to become less complex relative to the normal state.

\section{Conclusion}

EEG signal can be used as a reliable indicator of the state of the mind. Ever since the birth of 'nonlinear science' chaoticians of physiology, biomedical engineering and theoretical biology are searching for meaningful chaotic parameters in physiological processes. Here we have evaluated linear parameters and nonlinear parameters: $C D$, LLE, ApEn and Hurst Exponent. The results of these were subjected to ANOVA test with more than $90 \%$ confidence interval giving excellent ' $p$ ' values in all cases. The importance of ApEn lies in the fact that it is measure of the disorder in the EEG signal. This ApEn falls indicating the decrease in the disorder of the EEG signal due to rock/classical music and reflexology. This indicates that, the $\propto$ wave tends to become more predominant due to the influence of the music and reflexology. Similarly, Correlation Dimension, LLE and Hurst Exponent decreases due to the music and reflexology. In this work, we have proposed a set of ranges for these nonlinear parameters for various mental states.

\section{Authors' Contributions}

Thelma $\mathrm{T}$ and Fadilah A carried out the software implementation. Kannathal $\mathrm{N}$ and Rajendra U Acharya carried 
out the design, analysis and coordination of the work Sadasivan K Puthusserypady conceived of the study and participated in its design and coordination. All authors read and approved the final manuscript.

\section{References}

I. Stein KM, Lippman N, Kligfield P: Fractal rhythms of the heart Journal of Electrocardiology 1992, 24:72-76.

2. Hoyer D, Schmidt K, Bauer R, Zwiener U, Kohler M, Luthke B, Eiselt $M$ : Nonlinear analysis of heart rate and respiratory dynamics. IEEE Engineering in Medicine and Biology Magazine 1997, 16(I):31-39.

3. Kaplan DK, Cohen JR: Searching for Chaos in fibrillation. Annals NY Academic Science 1991:367-374.

4. Duke W, Pritchard WS, Krieble KK: Dimensional analysis of resting human EEG II: Surrogate data testing indicates nonlinearity but not low-dimensional chaos. Psychophysiology 1995 32:486-491.

5. Boccaletti S, Grebogi C, Lai YC, Mancini H, Mazaet D: The control of chaos: Theory and applications. Physics Reports 2000, 329: 108-109.

6. Glass L, Michel RG, Mackey M, Shrier A: Chaos in neurobiology. IEEE Trans Sys Man \& Cybernatics 1983, I3(5):790-798.

7. Jaeseung J, Jeong-Ho C, Kim SY, Seol-Heui H: Nonlinear dynamica analysis of the EEG in patients with Alzheimer's disease and vacular dementia. Clin Neurophysiol 200I, 18(I):58-67.

8. Philippe F, Henri K: Is there chaos in the brain? Concepts of nonlinear dynamics and methods of investigation. Life Sciences 200I, 324:773-793.

9. Babloyantz A: Evidence of chaotic dynamics during sleep cycle. In Dimensions and entropies in Chaotic system. Edited by: Mayer-Kress G. Berlin: Springer-Verlag; 1986.

10. Bessar E: Biophysical and physiological systems analysis. London: Addison-Wesley; 1960.

II. Mayer G, Layne SC: Dimensionality of the human encephalogram. In perspectives in Biological Dynamics and Theoretical Medicine. Annals NY Academic Science 1987:504-507.

12. Rapp PE, Bashore T, Martinerie J, Albano A, Zimmerman I, Mess A Dynamics of brain electrical activity. Brain Topography 1989, 2:99-118.

13. Rapp PE: Chaos in the neurosciences: cautionary tales from the frontier. Biologist 1993, 40:89-94.

14. Freeman W]: A proposed name for aperiodic brain activity: stochastic chaos. Neural Networks 2000, 13: I I-13.

15. Wright J], Liley DTJ: Dynamics of the brain at global and microscopic scales. Neural networks and the EEG. Behavioral and Brain Sciences 1996, 19:285-320.

16. Pijn JPM: Quantitative evaluation of EEG signals in epilepsy: nonlinear association time delays and nonlinear dynamics. Ph.D. thesis University of Amsterdam; 1990.

17. Pijn JP, Velis DN, van der Heyden MJ, DeGoede J, van Veelen CW, Lopes da Silva FH: Nonlinear dynamics of epileptic seizures on basis of intracranial EEG recordings. Brain Topography 1997, 9:249-270.

18. Rombouts SARB, Keunen RWM, Stam CJ: Investigation of nonlinear structure in multichannel EEG. Phys Lett A 1995 , 202:352-358.

19. Lamberts J, Van den Broek PLC, Bener J, Van Egmond J, Dirksen R, Cohen AML: Correlation dimension of the human electroencephalogram corresponding to cognitive load. Neuropsychobiology 2000, 4 I (3): I 149-I 53.

20. Bradley E: Time-series analysis. Intelligent data analysis: An introduction. Edited by: Berthold M, Hand D. Berlin: Springer-Verlag; 1999.

21. Theiler J: Spurious dimension from correlation algorithms applied to limited time-series data. Phys $\operatorname{Rev} A$ 1986, 34:2427-2432

22. Theiler J, Eubank S, Longtin A, Galdrikian B, Farmer JD: Testing for nonlinearity in time series: the method of surrogate data. Physica D 1992, 58:77-94.

23. Burioka N, Cornelissen G, Halberg F, Kaplan D T, Suyama H, Sako T, Shimizu E: Approximate entropy of human respiratory movement during eye-closed waking and different sleep stages. Chest 2003, I 23:80-86.

24. Bipoac Owners Guide. 200I
25. Voss RF, Clarke J: I/f noise in music and speech. Nature 1975, 258:3|7-3I8.

26. Fraser AM, Swinney HL: Independent coordinates for strange attractors from mutual information. Phys Rev A 1986, 33: II34- II 40.

27. Fraser AM: Information and entropy in strange attractors. IEEE transactions on Information Theory 1989, 35:245-262.

28. Grassberger P, Procaccia I: Characterization of strange attractors. Phys Rev Lett 1983, 50:346-349.

29. Grassberger P, Procaccia I: Measuring the strangeness of strange attractors. Physica D 1983, 9:189-208.

30. Packard NH, Crutchfield JP, Farmer JD, Shaw RS: Geometry from a time series. Phys Rev Lett 1980, 45:7|2-716.

31. Takens F: Detecting Strange Attractors in turbulence. In Dynamical Systems and Turbulence Berlin: Springer; 1981.

32. Sauer T, Yorke J, Casdagli M: Embedology. Journal of Statistical Physics 1994, 65:579-616.

33. CDA User Manual. 200I.

34. Theiler J: Spurious dimensions from correlation algorithms applied to limited time-series data. Phys Rev A 1986, 34:2427-2432.

35. Inouye T, Shinosaki K: Quantification of EEG irregularity by use of the entropy of the power spectrum. Electroencephalogr Clin Neurophysiol I99I, 79(3):204-210.

36. Fell J, Roschke J: A comparison between spectral and nonlinear EEG measures. Electroencephalogr Clin Neurophysiol 1996, 98(5):40I -410

37. Amari S: Differential-Geometrical methods in statistics. New York: Springer-Verlag; 1985.

38. Grassberger $P$, Schrieber $T$ : Nonlinear time sequence analysis. Int J Bifurcation and Chaos 199I, I(3):5।2-547.

39. Gonzalez Andino SL, de Peralta Menendez Grave: Measuring the complexity of time series: an application to neurophysiological signals. Hum Brain Mapp 2000, I I ( I):46-57.

40. Quiroga RQ, Arnhold J, Lehnertz K, Grassberger P: Kulback-Leibler and renormalized entropies: applications to electroencephalograms of epilepsy patients. Stat Phys Plasmas Fluids Relat Interdiscip Topics Volume 62. ; 2000:8380-8386.

41. Pincus SM: Approximate entropy as a measure of system complexity. Proc Natl Acad Sci USA 1991, 88:2297-230I.

42. Pincus SM, Goldberger AL: Physiological time-series analysis: what does regularity quantify? Am J Physiol 1994, 266:HI643-HI656.

43. Bruhn J, Ropcke H: Approximate entropy as an electroencephalographic measure of anesthetic drug effect during desflurane anesthesia. Anesthesiology 2000, 92(3):715-26.

44. Steyn-Ross ML, Steyn-Ross DA, Sleigh JW, Liley DT: Theoretical electroencephalogram stationary spectrum for a whitenoise-driven cortex: evidence for a general anestheticinduced phase transition. Phys Rev E 1999, 60(6):7299-73। I.

45. Dangel S, Meier PF, Moser HR, Plibersek S, Shen Y: Time series analysis of sleep EEG. Computer assisted Physics 1999:93-95.

46. Wolf A, Swift JB, Swinney LH, Vastano JA: Determining Lyapunov exponent from a time series. Physica $D$ 1985, 16:285-317.

47. Das A, Das P, Roy AB: Applicability of Lyapunov Exponent in EEG Data Analysis. Complexity International 2002.

48. Theiler J, Eubank S, Longtin A, Galdrikian B, Farmer JD: Testing for nonlinearity in time series: the method of surrogate data. Physica D 1992, 58:77-94.

49. Bruce JW: Fractal Physiology and Chaos in Medicine. World Scientific Press; 1990

50. Battacharya J: Complexity analysis of spontaneous EEG. Acto Neurobiol Exp 2000, 60:495-501.

5I. Klonowski W, Jernajczyk W, Niedzielska K, Rydz A, Stepien R Quantitative measure of complexity of EEG signal dynamics. Acta Neurobiol Exp (Wars) 1999, 59:315-321.

52. Palus M, Dvorak I, David I: Remarks on spatial and temporal dynamics of EEG. In: Mathematical approaches to brain functioning diagnostics Edited by: Dvorak I, Holden AV. Manchester University Press; 1991:369-385

53. Soong $\mathrm{AC}$, Stuart $\mathrm{Cl}$ : Evidence of chaotic dynamics underlying the human alpha-rhythm electroencephalogram. Biol Cybern $1989,62: 55-62$

54. Stam CJ, Pijn JPM, Suffczynski P, Lopes da Silva FH: Dynamics of the human alpha rhythm: evidence for non-linearity? Clin Neurophysiol 1999, I I 0: 180 I-18I3. 
55. Szelenberger W, Wackermann J, Skalski M, Niemcewicz S, Drojewski $\mathrm{J}$ : Analysis of complexity of EEG during sleep. Acta Neurobiol Exp (Wars) 1996, 56:165-169.

Publish with Bio Med Central and every scientist can read your work free of charge

"BioMed Central will be the most significant development for disseminating the results of biomedical research in our lifetime. " Sir Paul Nurse, Cancer Research UK

Your research papers will be:

- available free of charge to the entire biomedical community

- peer reviewed and published immediately upon acceptance

- cited in PubMed and archived on PubMed Central

- yours - you keep the copyright

Submit your manuscript here:

http://www.biomedcentral.com/info/publishing_adv.asp
BioMedcentral 